displacement results. But as the will is the dominant $\mid$ many seem to think that with a given specific remedy power of the mind and owes its strength to support for any given disease, all that is necessary is to apply from the other faculties, it is evident that its weak- that remedy mechanically, without respect to anyness is due to a general loss of energy, which sub- thing else, physiologic or pathologic. Chronic tuserves the mind as a whole, and as a consequence thus becomes an added cause for farther evil.

The evidence of a delusion is not always readily recognized, even by asylum physicians. Some subjects secrete their delusions; some have their delusions instantly dissipated by the presence of a physician, officer or stranger; some only have momentary delusions or perhaps frequently changed; some change with prominent events; some have delusions so buried that they can only be elicited by particular conditions and requiring the analytical skill of an expert.

But the point of present issue is that where there is no delusion, there is no insunity, for the mind that is competent to comprehend facts and their bearings within the scope of its education and the limits of ordinary surroundings is a mind capable of correction on any error; and, conversely, a mind that is not thus competent must of necessity beget delusion of one form or another which it is as incompetent to discharge, no matter what the evidence combating.

\title{
FURTHER REPORT OF THE SERLMTHERAPY IN TUBERCULOSIS.
}

BY PAUL PAQUIN, M.D.

IHYSICIAN-IN-CHIEF ST. LOUIS SANITARILM FOR THROAT AND LLNG DISEASES.

$$
\text { sT. LouIs, yo. }
$$

Up to this date all of the reports which I have had the honor to make to the medical profession, bearing on the use of the horse-blood serum in the treatment of tuberculosis, cover a number of cases in which the improvement had been more or less marked. Our experiments up to the last report had not gone far enough to justify the statement that we had, in fact, cases of tuberculosis which had been cured. The present writing is chiefly for the purpose of reporting results of a more positive nature and, at the same time, to say a few words on the complications that may arise during serum injections; the conditions which militate against its use; also the extent, so far as I am able to present from past experience, of the expectations that may be based on the new treatment. I will begin with the latter propositions.

In the first place, one should always bear in mind that tuberculosis varies a great deal with individuals and with respect to the organs affected. Pulmonary tuberculosis itself presents a wide range of pathologic conditions that must be judged and interpreted according to the extent and nature of each, locally and generally on the system. Consequently, no two cases of pulmonary consumption can, with safety, and hope of success, be treated alike by any one remedy, irrespective of the general and local constitutional or organ disturbances. One would suppose that a specific treatment should apply to all forms of it. One would think that if creasote is good in one case it ought to be in another. If inhalation of essential oils does good in one case it should benefit in another. If serum injections produce good results in a given case, they should have similar effects, irrespective of any other treatment, in any case, affecting any organ.

But such is not the case. It is unfortunate that so tubercles th of them), each surrounded by a special envelope, impenetrable by the circulation or by medicines. These tubercles may be surrounded by acute inflammatory processes or free from them. Some may be in an advanced stage and others just forming. In such cases, the opening of the tubercles happens gradually and successively; that is, some of them break to-day and others to-morrow or later on. Consequently, a more or less constant process of eruption goes on for months or years. Sometimes eventually, cavities are formed, during which certain principles of the morbid matter are partly absorbed, but mostly expelled by expectoration. It is evident that no form of treatment can arrest these slow cases promptly. The good results of serumtherapy in these have been slow in proportion to the nature of the affection, but have occurred, and we have under observation patients of this class who are unquestionably steadily improving, if we are to judge by the disappearance of the chief symptoms, such as night sweats, fever, loss of appetite, emaciation. Indeed, to arrest the progress of the disease in such cases is itself progress toward a cure. In another form of tuberculosis, one may meet with contracted lungs, sometimes with alterations of a more or less fibrous character, lungs encased usually in a narrow or otherwise defective chest. These cases are also very slow (they do not always end with cavities), and serumtherapy as well as other agents must naturally fail to bring quick improvement.

It is only slowly that one can reach such patholugic lesions, whatever agent is used.

On the other hand, we find chronic cases of tuberculous processes existing here and there, or localized in one apex or both apices, which are of a subacute character, and around which there exists more or less infiltration which, under physical examination, simulate to some degree the fibrous consolidations which I have mentioned before. This form of tuberculosis yields more readily to the serumtherapy than those above mentioned.

Again, there are cases of chronic tuberculosis of the lungs in which the tuberculous alterations con. sist first in localized inflammatory processes, unknown sometimes to the individual until a hemorrhage takes place and which sometimes end in cavities more or less extensive, and more or less serious according to their location, etc. One occasionally meets with such cases without detecting any other alterations in the lungs.

It must be apparent to any physician that the various conditions just mentioned (which do not by any means cover all the types of pulmonary phthisis) are not to be treated by any stereotyped formula and serotherapy, acting as a specific agent, should not be depended upon exclusively, irrespective of other conditions in the diseased organization. In other words, every case should be managed scientifically, using the serum only for the purpose it is intended, namely, to give to the organism the physiologic elements which it lacks to fight the tuberculous process from within.

Most of the chronic forms are mixed infections, 
and therefore demand additional treatment, and were they not such, accessory therapy is certainly in order to meet any special condition, and physicians should not neglect them under any circumstances. In our first reports we made it a point to explain that all our experimental cases had been treated exclusively with serum. Exclusive serumtherapy was - necessary in those experiments, because we had to demonstrate as far as possible what the serum alone could do, but we are not justified in this early stage of the serumtherapy to lay aside everything else that may be useful to meet any special phase of disorders in a tuberculous individual. In fact, it is unscientific to treat diseases by any stereotyped method. Each case of any given disease should be considered on its merits, if I may so speak, and after proper consideration one should use the most potent or most specitic therapy and add to it, if necessary, according to what the symptoms may suggest.

We have beside to deal with acute forms of tuberculosis which, under ordinary treatment, may be classed as hopeless from the very beginning. It may be said that they always end in death. Medicine of the past shows no authentic records of cures in any such case. It is in these, however, that serumtherapy has proved its efficacy most strikingly. It is only within the last three months that we have had opportanities of testing the serum in this form of the disease and, already, I am in position to point to three patients in which the diagnosis made microscopically and physically was unmistakable, who have arisen from a bed of sickness where everything pointed to death within a short time. These cases, were not in my own practice, and, consequently, I am not at liberty at present to give their history. But I will mention that I have seen two of them, and that I have verified the microscopic analyses previously made in them and know that both of them had acute tuberculosis of the lungs in the worst form and both are now entirely free from bacilli, and are up and ahout and gaining strength, and one has absolutely no physical or microscopic sign of the disease left. This one, a young lady of about 18 years, had been at the time of the beginning of the treatment, prostrated in bed some four or five weeks; had had a temperature of 104 to 105 during three weeks preceding the injections; had had cerebral symptoms to the degree of absolute loss of mind during all that time; had both lungs so seriously involved that breathing was painfully short and laborious; emaciation and weakness were so pronounced that she was abso. lutely unable to raise her head, and could barely lift her hand. She was treated about three weeks with the serum and the temperature went down steadily after about four days from 105 to 98.5 ; the lungs gradually but steadily cleared up; hemoptysis and expectoration ceased; the bacilli entirely disappeared; the clouds were lifted from the mind; the appetite returned and the young lady is out of bed, up and around, and putting on flesh rapidly. This is the most striking result we have obtained by the exclusive use of the serum. The physician in charge, who will report the case himself shortly, excluded all other medicines because the case, after consultation with other physicians, was considered hopeless by any of the old modes of therapy. The serum was used as the last resort without any hope whatever.

The other patient of the two just mentioned, whom I have seen, was an old gentleman who had also been prostrated in bed several wer zs with acute tuberculosis diagnosed as such by some of the most eminent physicians of St. Louis, who, after consultation, considered his case hopeless. This patient began the serumtherapy under another physician, and within six weeks, I believe, was also raised from his bed and is out and about. Not a bacillus is to be found in his expectoration to-day; one lung is entirely cleared, but in the other there remain some adhesions, which will probably remain, as they seem to be of a fibrous character. Certainly the tuberculous phenomena seem to have been arrested; the germs are there no more, and the ravages which they have produced are being healed.' We have also two cases of chronic tuberculosis without any more symptoms of the disease except the evidence of the cicatrix. They were both bedridden and are now both working hard every day.

Now as to the complications that may arise in acute tuberculosis by injections of serum, I will mention first the general disturbances. Constant injections of serum may result in rheumatic symptoms, partic. ularly in those with rheumatic diathesis. Swelling and pain of the articulations and pain in the muscles may arise. By suspending injections and using lithia the symptoms disappear. Occasionally urticaria results, scattered over the skin around the ankles, body, neck, etc. A curious accident that occurs sometimes is a sudden disturbance of the circulation, evidenced by a very pronounced flushing of the face, a condition which usually promptly disappears. This occurs during injections or just after. The face becomes gorged with blood, the patient feels dizzy and will fall if not assisted to lie down. These symptoms appear and disappear within two to five minutes, in my experience. Two or three times, after such occurrences, there resulted a chill and occasionally a headache, and pain appeared in the spinal column. In no instance, except one, did the heart suffer during the trouble. In this case it became very feeble and hypodermic injections of stimulants (whisky and nitro-glycerin) were deemed useful to assist recuperation. This disturbance of the circulation occurred in my practice and my experiments eight times in a total of some eight thousand injections. Whether it can be fatal or not, I am unable to say, but it is so rare that it is not to be considered in any worse light than any untoward symptom that may arise from the administering of any other medicine of a potent character which we use daily in medical practice, such as cocain, sulfonal and numerous other medicinal agents which the readers will readily recall to memory.

With respect to the kidney's action, I have to mention that in two cases in which there existed already a trace of albumin, albuminuria increased without, however, producing any of the serious symp. toms that we note in Bright's disease. In neither of these cases were there any casts to be found. In another case, which was not directly under my control, and which unfortunately had not been analyzed before beginning the injections, albumin was found in great quantities after the patient had been treated two or three weeks. I am unable to say whether this came from the injections or not, inasmuch as it occurs frequently enough, that albuminuria exists with tuberculosis. On this point, I will mention that,

1 This old gentleman patient now weighs nearly 200 pounds. Weight at beginning of treatment less than 160 . 
being in the habit of analyzing the urine of every case of tuberculosis before treatment, I have found the last four months, six cases of albuminuria in thirty-two cases of pulmonary consumption.

From the foregoing, I judge that it is not wise to use the serum in cases where there is kidney inactivity. Locally, the serum injections may produce erythema and local irritations more or less pronounced. These are sometimes sufficient to produce an elevation of temperature and they should be treated according to their nature. It would seem from the foregoing, that serum injections in tuberculosis, or in any other disease for that matter, should not be considered entirely free from possible complications, and that every case treated should first be thoroughly analyzed and treated on its merits. With respect to the results of the serum in the hands of others, I am pleased to say that I am getting daily excellent reports which will serve materially in deciphering the conditions in which the serum will or will not act beneficially or will be too slow for practical purposes. I have now in my hands, reports covering the use of the serum in various parts of the country in many different tuberculous conditions from which I shall glean material for an article explaining various kinds of results. All these, I can now state conscientiously, give evidence that the principle of serotherapy in consumption is correctly interpreted in our experiments and our judgment as made known by our reports. In New Orleans, California, Missouri, Illinois, etc., we have had excellent results, and, naturally a percentage of failures.

Before closing, I wish to state that in local tuberculosis of the skin, glands, and surgical cases with fistulæ, with and without operations, the results have been positively beneficial. Specific reports will be given by the physicians who have undertaken such cases in this city and elsewhere. Cures have practically occurred.

In conclusion, I beg to warn the over-anxious against the idea that a few weeks of treatment of any case of chronic tuberculosis will effect a cure. The cases we can now report as free from tuberculous symptoms and which were in the first and second stages of the disease have been treated not less than four to six months steadily. Four to ten months of steady treatment should be contemplated from the onset. No miraculous or magic effects should be expected by any doctor or patient. Considering that tuberculosis is fatal in 99 per cent. of cases, and generally very slow, this conclusion is entirely reasonable.

\section{SOCIETY PROCEEDINGS.}

\section{American Public Health Association.}

\section{Proceedings of the Twenty-Third Annual Meeting held in Den- ver, Colo., Oct. 1-4, 1895. \\ Second Day-Wednesdy, October 2. (Continued from $p$. 682.)}

The Association reconvened at 10 o'clock, A.m.in the Brown Palace Hotel, with President Bailey presiding.

There was a larger attendance than during the preceding day's sessions, as a number of delegates had arrived since last adjournment.

Chairman Sewall, of the Local Committee of Arrangements made announcements regarding various trips, excursion rates to same; social functions, etc.

The Executive Committee, through Secretary Watson, recommended that the names of the applicants in his hands be elected to membership. Upon motion duly seconded the Secretary cast the ballot of the Association for them.

The Committee also recommended the adoption of the resolutions offered at Tuesday morning's session by Dr. Mitchell, regarding the transmission through the mails of bacteriologic specimens when properly prepared so that no danger could possibly accrue to those handling the packages, etc. Dr. Mitchell then exhibited two samples of tubes and cases that he had devised for this purpose which he had proved was perfectly safe to send specimens in, and deemed to be absolutely safe to admit through the mails.

The resolutions were then unanimously adopted. The committee also recommended that the committee on the centennial celebration of the discovery of vaccination be restored by the Association. Adopted.

The committee recommended the creation of a new committee, namely, a Committee on Forestry and its Relation to Public Health. Adopted.

After some additional routine business had been disposed of, Treasurer Henry D. Holton, of Brattleboro, Vt., submitted his annual report which showed receipts from September, 1894 , to date from all sources to be $\$ 3,627$, and disbursements as per fourteen vouchers; that there is remaining in the treasury $\$ 493.98$, Index fund unexpended. Upon motion, the report was received and referred to an auditing committee corsisting of Mr. Crosby Gray, Dr. H. B. Horlbeck, and Dr. J. N. MeCormack.

Dr. C. N. HewitT introduced the following resolution on the death of M. Louis Pasteur, which was duly seconded :

Resolved, That this Association has heard with profound sorrow of the death of Louis Pasteur. That it offers to his family, to his assistants at the Institute Pasteur, to his country, to the members of the profession, sincere condolence in the loss of a master of science. His work for the alleviation of human suffering is as he would have chosen-his everlasting monument. His example as an humble and at. tentive servant and student of nature, is the precious legacy he has left us.

On motion of Dr. McCormack, a silent rising vote was taken. In addition to the foregoing, upon motion of Dr. Formento, a cablegram was sent to Dr. Roux, collaborateur to Pasteur, containing these words: "The American Public Health Association mourns the loss of Pasteur."

The roll of the Advisory Council was called, and the following members were present:

Colorado-Dr. Henry Sewall, Denver.

Connecticut-Dr. R. S. Goodwin, Thomaston.

Delaware-W. C. R. Calquohoven, Wilmington.

Illinois-Dr. Liston H. Montgomery, Chicago.

Indiana-Dr. I. S. Whitesides, Franklin.

Iowa-Dr. A. W. Cantwell, Daven port.

Kansas, Dr. Daniel C. Jones.

Kentucky-Dr. J. N. McCormack, Bowling Green.

Louisiana-Dr. Felix Formento, New Orleans.

Maine-Dr. A. G. Young, Augusta.

Maryland-Dr. James $F$. MeShane, Baltimore.

Massachusetts-Dr. S. H. Durgin, Boston.

Michigan-Dr. Cressy L. Wilbur, Lansing.

Minnesota-Dr. C. N. Hewitt, Minneapolis.

New Hampshire-Dr. Granite P. Conn, Concord.

New Jersey-Dr. Henry Mitchell, Asbury Park.

New Mexico-Dr. S. D. Swope, Deming.

New York-Dr. A. W. Suiter, Herkimer.

North Carolina-Dr. R. H. I,ewis, Raleigh.

North Dakota-Dr. John Montgomery.

Ohio-Dr. Charles O. Probst, Columbus.

Pennsylvania-Crosby Gray, Pittsburg.

Rhode Island-Dr. Gardner T. Swartz, Providence.

South Carolina-Dr. H. B. Horlbeck, Charleston.

Tennessee-Dr. W. C. Bailey, Knoxville.

Vermont-Dr. H. D. Holton, Brattleboro.

Wisconsin-Dr. U. O. B. Wingate, Milwaukee.

District of Columbia-Dr. Ralph Walsh, Washington.

U. S. Army-Deputy Surgeon-General A. H. Woodhull, Denver, Colo.

U. S. M.-H. Service-Surgeon P. H. Bailbache, New York. U. S. Navy-Surgeon Bates, New York.

Dominion of Canada-Dr. F. Montizambert, Quebec.

Province of Ontario-Dr. Peter H. Bryce, Toronto.

Province of Quebec-Dr. E. P. La Chapelle, Montreal.

Manitoba-.-Dr. James Patterson, Winnipeg.

New Brunswick-Dr. George E. Coulthard, Fredericton.

The appointment of Mexican members to the Council was deferred until the arrival of the delegates from the Republic.

Dr. H. B. HorLBECK, Health Officer of Charleston, S. C.. read the first paper which was a brief one, entitled "Munici- 